\title{
Laplace Inverse Transform for Functions of Type $n$th Root of a Product of Linear Factors
}

\author{
Uriel Salmeron-Rodriguez \\ Centro de Ingenieria y Desarrollo Industrial (CIDESI), Queretaro, Mexico \\ Email: ur_18_@hotmail.com
}

How to cite this paper: Salmeron-Rodriguez, U. (2017) Laplace Inverse Transform for Functions of Type $n$th Root of a Product of Linear Factors. Open Access Library Journal, 4: e3741.

https://doi.org/10.4236/oalib.1103741

Received: June 13, 2017

Accepted: July 3, 2017

Published: July 6, 2017

Copyright (๐ 2017 by author and Open Access Library Inc.

This work is licensed under the Creative Commons Attribution International License (CC BY 4.0).

http://creativecommons.org/licenses/by/4.0/

\section{(c) (i) Open Access}

\begin{abstract}
In this work, we present four results for the Laplace inverse transform of functions that involve the $n$th root of a product of linear factors. In order to find the Laplace inverse transform, we considered a branch cut for the $n$th root and a region of suitable integration, to avoid the branching points. Due to that, the solution is in terms of integrals, we easily approach this solution for some specific parameters.
\end{abstract}

\section{Subject Areas}

Mathematical Analysis, Mathematical Logic and Foundation of Mathematics

\section{Keywords}

Laplace Inverse Transform, Multivalued Functions, Integration Contour, Stehfest Numerical Method

\section{Introduction}

Modeling phenomena using partial differential equations are attractive to physicists, mathematicians, engineers, etc., this is due to many phenomena such as diffusion of particles, heat diffusion, fluid flow behavior on porous media, among others, are modeled with this type of differential equations (see [1]-[7]). However, when we use the Laplace transform to find the solution of these models, it is likely to find multivalued functions, so the Laplace inverse transform is commonly solved by numerical methods, for example, in [8] [9] studied models that predict the fluid flow on porous media, they used the Laplace transform and found multivalued functions of the type $\sqrt{ }_{\text {, }}$, and to give the solution through Laplace inverse transform they used the Stehfest numerical method. The Stehfest numerical method has restrictions for functions that have discontinuities [10], so it is more advisable to find the exact solution. In some 
cases, it is possible to give the exact solution [11], but to obtain the exact solution of these models, a deeper study of the Laplace inverse transform of the multivalued functions found is necessary. In this work, we study Laplace inverse transform for functions that involve the $n$th root of a product of linear factors. It is divided into two sections as follows: In the first section, we use the Laplace inverse transform defined as

$$
f(t)=\frac{1}{2 \pi i} \int_{\delta-i \infty}^{\delta+i \infty} \mathrm{e}^{s t} F(s) \mathrm{d} s,
$$

to find the function $f(t)$. Here $\delta>0$ and $F(s)$ involve to

$$
\sqrt[n]{\left(s+a_{0}\right)\left(s+a_{1}\right)\left(s+a_{2}\right) \cdots\left(s+a_{k-1}\right)\left(s+a_{k}\right)} \text {, }
$$

where $n$ is positive integer and $a_{0}<a_{1}<a_{2}<\cdots<a_{k}$ are real positive. The function $f(t)$ is represented in terms of integrals that are easily approximated numerically. To find this function, we consider the branch cut and the integration contour of Figure 1.

In section two, We give analytical examples and also numerically solve the function $f(t)$ for some particular cases and compare versus the Stehfest

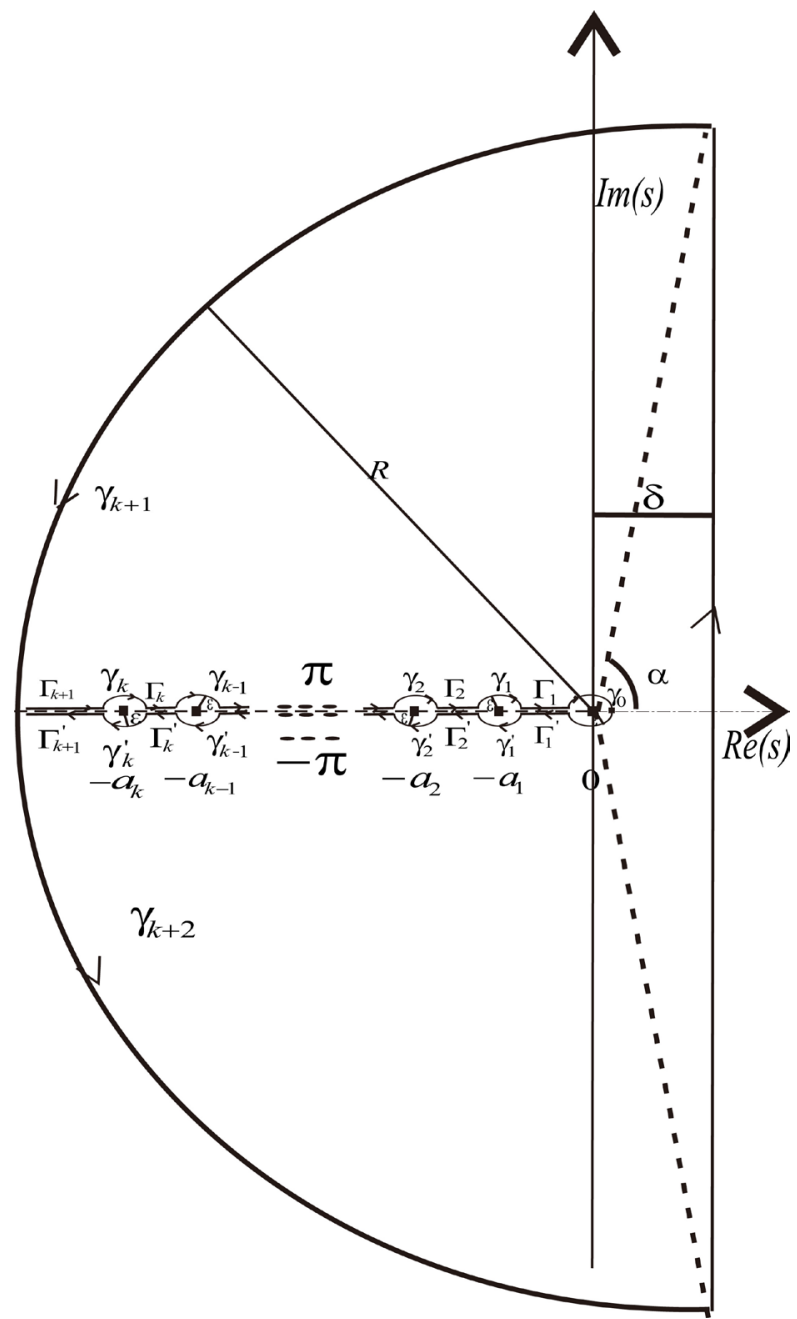

Figure 1. Integration contour. 
numerical method. We also show that the Stehfest numerical method does not approximate well to the exact solution near the discontinuities.

\section{Theorems}

In this section, we propose and prove four theorems associated with the Laplace

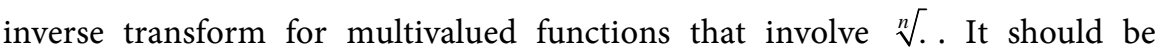
mentioned that in particular Theorem 4 can be used to find the solution of some differential equations that model the fluid flow on porous media, for example [11].

Theorem 1. Suppose $n \in \mathbb{Z}^{+}, t>0,0=a_{0}<a_{1}<a_{2}<\cdots<a_{k}<a_{k+1}=\infty$ and

$$
F(s)=\frac{1}{s} \sqrt[n]{\left(s+a_{0}\right)\left(s+a_{1}\right)\left(s+a_{2}\right) \cdots\left(s+a_{k-1}\right)\left(s+a_{k}\right)}
$$

then the Laplace inverse transform of the function $F(s)$ is given by

$$
f(t)=\frac{1}{\pi} \sum_{j=0}^{k} \sin \left(\frac{(j+1) \pi}{n}\right) \int_{a_{j}}^{a_{j+1}} \frac{1}{x} \mathrm{e}^{-x t} u_{j}(x) \mathrm{d} x
$$

where

$$
u_{j}(x)=\sqrt[n]{\left(x-a_{0}\right)\left(x-a_{1}\right) \cdots\left(x-a_{j}\right)\left(-x+a_{j+1}\right) \cdots\left(-x+a_{k-1}\right)\left(-x+a_{k}\right)} .
$$

Proof. Using the Laplace inverse transform, we have

$$
f(t)=\frac{1}{2 \pi i} \int_{\delta-i \infty}^{\delta+i \infty} \mathrm{e}^{s x} F(s) \mathrm{d} s
$$

As $a_{0},-a_{1},-a_{2}, \cdots,-a_{k}$ are branch points of the function $F(s)$, then we consider the region of the Figure 1 and the branch cut $-\pi<\operatorname{Arg}(s)<\pi$ for the $n$th root. Using Cauchy Theorem is found

$$
\begin{aligned}
f(t)= & \frac{1}{2 \pi i} \int_{\delta-i \infty}^{\delta+i \infty} \mathrm{e}^{s x} F(s) \mathrm{d} s \\
= & -\frac{1}{2 \pi i}\left(\int_{\Gamma_{1}}+\int_{\Gamma_{1}^{\prime}}+\cdots+\int_{\Gamma_{k+1}}+\int_{\Gamma_{k+1}^{\prime}}+\int_{\gamma_{0}}\right) \\
& -\frac{1}{2 \pi i}\left(\int_{\gamma_{1}}+\int_{\gamma_{1}^{\prime}}+\cdots+\int_{\gamma_{k}}+\int_{\gamma_{k}^{\prime}}+\int_{\gamma_{k+1}}+\int_{\gamma_{k+2}}\right) .
\end{aligned}
$$

It is easy to prove that $\int_{\gamma_{0}}=0$ when $\epsilon \rightarrow 0$. In addition also $\int_{\gamma_{j}} \rightarrow 0$ to $j=1,2, \cdots, k$ when $\epsilon \rightarrow 0$, this is because if $s=-a_{j}+\epsilon \mathrm{e}^{i \theta}$ with $0<\theta<\pi$ then

$$
\left|\int_{\gamma_{j}}\right|<\int_{0}^{\pi} \frac{\mathrm{e}^{\left(-a_{j}+\epsilon\right) t}}{\left|-a_{j}+\epsilon\right|} \sqrt[n]{\left(-a_{j}+\epsilon+a_{0}\right) \cdots\left(-a_{j}+\epsilon+a_{j}\right) \cdots\left(-a_{j}+\epsilon+a_{k}\right)}(\epsilon) \mathrm{d} \theta,
$$

thus $\int_{\gamma_{j}} \rightarrow 0$ when $\epsilon \rightarrow 0$. Analogously if $s=-a_{j}+\epsilon \mathrm{e}^{i \theta}$ with $-\pi<\theta<0$ we prove that $\int_{\gamma_{j}^{\prime}} \rightarrow 0$ when $\epsilon \rightarrow 0$.

For $\int_{\gamma_{k+1}}$, we obtain

$$
\left|\int_{\gamma_{k+1}}\right|<\int_{\alpha}^{\pi} \mathrm{e}^{R \cos (\theta) t} \sqrt[n]{\left(R+a_{0}\right)\left(R+a_{1}\right) \cdots\left(R+a_{k}\right)} \mathrm{d} \theta
$$


as $t>0$ and $\cos \theta$ is negative in $(\pi / 2, \pi)$, then $\mathrm{e}^{R t \cos (\theta)} \rightarrow 0$ when $R \rightarrow \infty$, thus $\int_{\gamma_{k+1}} \rightarrow 0$ it is for $\delta$ small enough. Similarly for $\int_{\gamma_{k+2}}$.

For integrals over $\Gamma_{j+1}$ and over $\Gamma_{j+1}^{\prime}$ with $j=0,1,2, \cdots, k$ we analyze as follows: If $s=x \mathrm{e}^{i \pi}=-x$ with $x \in\left(a_{j}, a_{j+1}\right)$ we obtain

$$
\left(s+a_{k}\right)^{1 / n}= \begin{cases}\mathrm{e}^{\frac{i \pi}{n}}\left(x-a_{k}\right)^{1 / n} & \text { for } k=0,1, \cdots, j, \\ \mathrm{e}^{\frac{0 i}{n}}\left(-x+a_{k}\right)^{1 / n} & \text { for } k=j+1, j+2, \cdots, k .\end{cases}
$$

Using Equation (4) we find

$$
\begin{aligned}
u_{1}(x) & =\sqrt[n]{\left(s+a_{0}\right)\left(s+a_{1}\right) \cdots\left(s+a_{j}\right)\left(s+a_{j+1}\right) \cdots\left(s+a_{k}\right)} \\
& =(s)^{1 / n}\left(s+a_{1}\right)^{1 / n} \cdots\left(s+a_{j}\right)^{1 / n}\left(s+a_{j+1}\right)^{1 / n} \cdots\left(s+a_{k}\right)^{1 / n} \\
& =\mathrm{e}^{\frac{i \pi}{n}} x^{1 / n} \mathrm{e}^{\frac{i \pi}{n}}\left(x-a_{1}\right)^{1 / n} \cdots \mathrm{e}^{\frac{i \pi}{n}}\left(x-a_{j}\right)^{1 / n} \mathrm{e}^{\frac{i 0}{n}}\left(-x+a_{j+1}\right)^{1 / n} \cdots \mathrm{e}^{\frac{i 0}{n}}\left(-x+a_{k}\right)^{1 / n} \\
& =\mathrm{e}^{\frac{(j+1) i \pi}{n}} u_{j}(x),
\end{aligned}
$$

then

$$
\int_{\Gamma_{j+1}}=\int_{a_{j+1}}^{a_{j}} \frac{1}{X} \mathrm{e}^{-x t} u_{1}(x) \mathrm{d} x=-\int_{a_{j}}^{a_{j+1}} \frac{1}{x} \mathrm{e}^{-x t} u_{1}(x) \mathrm{d} x .
$$

On the other hand, if $s=x \mathrm{e}^{-i \pi}=-x$ con $x \in\left(a_{j}, a_{j+1}\right)$ we obtain

$$
u_{2}(x)=\sqrt[n]{\left(s+a_{0}\right)\left(s+a_{1}\right) \cdots\left(s+a_{j}\right)\left(s+a_{j+1}\right) \cdots\left(s+a_{k}\right)}=\mathrm{e}^{\frac{-(j+1) i \pi}{n}} u_{j}(x),
$$

then

$$
\int_{\Gamma_{j+1}^{\prime}}=\int_{a_{j}}^{a_{j+1}} \frac{1}{x} \mathrm{e}^{-x t} u_{2}(x) \mathrm{d} x .
$$

Thus, for Equation (5) and Equation (6) we find

$$
\int_{\Gamma_{j+1}}+\int_{\Gamma_{j+1}^{\prime}}=-2 i \sin \left(\frac{(j+1) \pi}{n}\right) \int_{a_{j}}^{a_{j+1}} \frac{1}{x} \mathrm{e}^{-x t} u_{j}(x) \mathrm{d} x .
$$

Finally, adding all integrals and replacing in Equation (3), we obtain

$$
f(t)=\frac{1}{\pi} \sum_{j=0}^{k} \sin \left(\frac{(j+1) \pi}{n}\right) \int_{a_{j}}^{a_{j+1}} \frac{1}{x} \mathrm{e}^{-x t} u_{j}(x) \mathrm{d} x
$$

Theorem 2. Suppose $n \in \mathbb{Z}^{+}, t>0,0=a_{0}<a_{1}<a_{2}<\cdots<a_{k}<a_{k+1}=\infty$ and

$$
F(s)=\frac{1}{\sqrt[n]{\left(s+a_{0}\right)\left(s+a_{1}\right)\left(s+a_{2}\right) \cdots\left(s+a_{k-1}\right)\left(s+a_{k}\right)}},
$$

then the Laplace inverse transform of the function $F(s)$ is given by

$$
f(t)=\frac{1}{\pi} \sum_{j=0}^{k} \sin \left(\frac{(j+1) \pi}{n}\right) \int_{a_{j}}^{a_{j+1}} \mathrm{e}^{-x t} u_{j}(x) \mathrm{d} x,
$$

where 


$$
u_{j}(x)=\frac{1}{\sqrt[n]{\left(x-a_{0}\right)\left(x-a_{1}\right) \cdots\left(x-a_{j}\right)\left(-x+a_{j+1}\right) \cdots\left(-x+a_{k-1}\right)\left(-x+a_{k}\right)}} .
$$

Proof. Again we use the region Figure 1. In analogy with the proof of the previous Theorem we have $\int_{\gamma_{0}} \rightarrow 0, \int_{\gamma_{j}} \rightarrow 0$ and $\int_{\gamma_{j}^{\prime}} \rightarrow 0$ when $\epsilon \rightarrow 0$ for $j=1,2, \cdots, k$ and also $\int_{\gamma_{k+1}} \rightarrow 0$ and $\int_{\gamma_{k+2}} \rightarrow 0$ when $R \rightarrow \infty$.

On the other hand we have

$$
\begin{gathered}
\int_{\Gamma_{j+1}}=\int_{a_{j+1}}^{a_{j}} e^{-x t} u_{1}(x)(-\mathrm{d} x)=\int_{a_{j}}^{a_{j+1}} \mathrm{e}^{-x t} u_{1}(x) \mathrm{d} x, \\
\int_{\Gamma_{j+1}^{\prime}}=-\int_{a_{j}}^{a_{j+1}} \mathrm{e}^{-x t} u_{2}(x) \mathrm{d} x,
\end{gathered}
$$

where

$$
u_{1}(x)=\mathrm{e}^{\frac{-(j+1) i \pi}{n}} u_{j}(x) \text { and } u_{2}(x)=\mathrm{e}^{\frac{(j+1) i \pi}{n}} u_{j}(x)
$$

then

$$
\int_{\Gamma_{j+1}}+\int_{\Gamma_{j+1}^{\prime}}=-2 i \sin \left(\frac{(j+1) \pi}{n}\right) \int_{a_{j}}^{a_{j+1}} \mathrm{e}^{-x t} u_{j}(x) \mathrm{d} x
$$

Therefore, we add all the integrals so we find the result. $\square$

Theorem 3. Suppose $n, m \in \mathbb{Z}^{+}, t>0,0=a_{0}<a_{1}<a_{2}<\cdots<a_{k}<a_{k+1}=\infty$ and

$$
F(s)=\frac{1}{s} \frac{\sqrt[n]{\left(s+a_{0}\right)\left(s+a_{2}\right)\left(s+a_{4}\right) \cdots\left(s+a_{k-3}\right)\left(s+a_{k-1}\right)}}{\sqrt[m]{\left(s+a_{1}\right)\left(s+a_{3}\right)\left(s+a_{5}\right) \cdots\left(s+a_{k-2}\right)\left(s+a_{k}\right)}},
$$

then the Laplace inverse transform of the function $F(s)$ is given by

$$
\begin{aligned}
f(t)= & \frac{1}{\pi} \sum_{j=0}^{\left[\frac{k}{2}\right]} \sin \left(\frac{(j+1) \pi}{n}-\frac{j \pi}{m}\right) \int_{a_{2 j}}^{a_{2 j+1}} \frac{1}{x} \mathrm{e}^{-x t} u_{2 j}(x) \mathrm{d} x \\
& +\frac{1}{\pi} \sum_{j=0}^{\left[\frac{k}{2}\right]} \sin \left(\frac{(j+1) \pi}{n}-\frac{(j+1) \pi}{m}\right) \int_{a_{2 j+1}}^{a_{2 j+2}} \frac{1}{x} \mathrm{e}^{-x t} u_{2 j+1}(x) \mathrm{d} x,
\end{aligned}
$$

where

$$
u_{j}(x)=\left(x-a_{0}\right)^{\frac{1}{n}}\left(x-a_{1}\right)^{-\frac{1}{m}} \cdots\left(x-a_{j}\right)^{\frac{1}{n}}\left(-x+a_{j+1}\right)^{-\frac{1}{m}} \cdots\left(-x+a_{k-1}\right)^{\frac{1}{n}}\left(-x+a_{k}\right)^{-\frac{1}{m}} .
$$

Note that $\left(x-a_{j}\right)^{1 / n}\left(-x+a_{j+1}\right)^{-1 / m}$ can change by $\left(x-a_{j}\right)^{-1 / m}\left(-x+a_{j+1}\right)^{1 / n}$ when $k$ is even or odd.

Proof. The proof is analogous to the previous theorems.

Theorem 4. Suppose $t>0, \quad r>0,0=a_{0}<a_{1}<a_{2}<\cdots<a_{k}<a_{k+1}=\infty$ and

$$
F(s)=\frac{1}{s} \frac{K_{0}\left(r \sqrt{\left(s+a_{0}\right)\left(s+a_{1}\right)\left(s+a_{2}\right) \cdots\left(s+a_{k-1}\right)\left(s+a_{k}\right)}\right)}{K_{0}\left(\sqrt{\left(s+a_{0}\right)\left(s+a_{1}\right)\left(s+a_{2}\right) \cdots\left(s+a_{k-1}\right)\left(s+a_{k}\right)}\right)},
$$

where $K_{0}$ is the modified Bessel function of order 0 then the Laplace inverse transform of the function $F(s)$ is given by 


$$
f(t)=1+\frac{1}{\pi} \sum_{j=1}^{\left[\frac{k}{2}\right]+1} \int_{a_{2 j-2}}^{a_{2 j-1}} \frac{1}{X} \mathrm{e}^{-x t} \Psi_{2 j-2}(r, x) \mathrm{d} x+\sum_{j=1}^{L} \int_{a_{2 j-1}}^{a_{2}} \frac{1}{X} \mathrm{e}^{-x t} \Psi_{2 j-1}(r, x) \mathrm{d} x
$$

where

$$
\begin{gathered}
L=\left\{\begin{array}{l}
{\left[\frac{k}{2}\right] \quad \text { for } k \text { even, }} \\
1+\left[\frac{k}{2}\right] \text { for } k \text { odd, }
\end{array}\right. \\
\Psi_{l}(r, x)= \begin{cases}\frac{I_{0}\left(u_{l}(x)\right) K_{0}\left(u_{l}(x) r\right)-K_{0}\left(u_{l}(x)\right) I_{0}\left(u_{l}(x) r\right)}{K_{0}\left(u_{l}(x)\right)^{2}+\pi^{2} I_{0}\left(u_{l}(x)\right)^{2}} & \text { for } l=2 j-1, \\
\frac{Y_{0}\left(u_{l}(x)\right) J_{0}\left(u_{l}(x) r\right)-J_{0}\left(u_{l}(x)\right) Y_{0}\left(u_{l}(x) r\right)}{J_{0}\left(u_{l}(x)\right)^{2}+Y_{0}\left(u_{l}(x)\right)^{2}} & \text { for } l=2 j-2,\end{cases}
\end{gathered}
$$

$I_{0}, Y_{0}, J_{0}$ are the corresponding Bessel functions of order 0 and

$$
u_{j}(x)=\sqrt{\left(x-a_{0}\right)\left(x-a_{1}\right) \cdots\left(x-a_{j}\right)\left(-x+a_{j+1}\right) \cdots\left(-x+a_{k-1}\right)\left(-x+a_{k}\right)} .
$$

Proof. For the proof, we used the following properties of the Bessel functions (see [12] [13] [14])

$$
\begin{gathered}
K_{v}(z)=i \frac{\pi}{2} \mathrm{e}^{\frac{\pi}{2} v i} H_{v}^{(1)}\left(z \mathrm{e}^{\frac{1}{2} \pi i}\right), \quad\left(-\pi<\arg z \leq \frac{1}{2} \pi\right), \\
K_{v}(z)=-i \frac{\pi}{2} \mathrm{e}^{-\frac{\pi}{2} v i} H_{-v}^{(2)}\left(z \mathrm{e}^{-\frac{1}{2} \pi i}\right), \quad\left(-\frac{1}{2} \pi<\arg z \leq \pi\right), \\
K_{v}\left(z \mathrm{e}^{i m \pi}\right)=\mathrm{e}^{-i m v \pi} K_{v}(z)-i \pi \sin (m v \pi) \csc (v \pi) I_{v}(z),(m \in \mathbb{Z}),
\end{gathered}
$$

where $H_{v}^{(1)}(z)$ and $H_{-v}^{(2)}(z)$ are the Hankel's functions of the order $v$.

Consider the case when $k$ is odd (for $k$ even the proof is analogous). Also consider the region of Figure 1 then $f(t)$ is like Equation (3). Then $\int_{\gamma_{j}} \rightarrow 0$, $\int_{\gamma_{j}^{\prime}} \rightarrow 0$ for $j=1,2, \cdots, k$ when $\epsilon \rightarrow 0$, also $\int_{\gamma_{0}}=-2 \pi i$ when $\epsilon \rightarrow 0$. For the integrals on $\Gamma_{j}$ and on $\Gamma_{j}^{\prime}$ with $j=1, \cdots, k+1$ we obtain the following: if $j$ is of the form $2 j-1$, we used Equation (10) and Equation (11), then

$$
\int_{\Gamma_{2 j-1}}+\int_{\Gamma_{2 j-1}^{\prime}}=-2 i \int_{a_{2 j-2}}^{a_{2 j-1}} \frac{1}{X} \mathrm{e}^{-x t} \Psi_{2 j-2}(r, x) \mathrm{d} x,
$$

where

$$
\Psi_{2 j-2}(r, x)=\frac{Y_{0}\left(u_{2 j-2}(x)\right) J_{0}\left(u_{2 j-2}(x) r\right)-J_{0}\left(u_{2 j-2}(x)\right) Y_{0}\left(u_{2 j-2}(x) r\right)}{J_{0}\left(u_{2 j-2}(x)\right)^{2}+Y_{0}\left(u_{2 j-2}(x)\right)^{2}} .
$$

On the other hand, if $j$ is of the form $2 j$, we used Equation (12) then

$$
\int_{\Gamma_{2 j}}+\int_{\Gamma_{2 j}^{\prime}}=-2 \pi i \int_{a_{2 j-1}}^{a_{2 j}} \frac{1}{X} \mathrm{e}^{-x t} \Psi_{2 j-1}(r, x) \mathrm{d} x,
$$

where

$$
\Psi_{2 j-1}(r, x)=\frac{I_{0}\left(u_{2 j-1}(x)\right) K_{0}\left(u_{2 j-1}(x) r\right)-K_{0}\left(u_{2 j-1}(x)\right) I_{0}\left(u_{2 j-1}(x) r\right)}{K_{0}\left(u_{2 j-1}(x)\right)^{2}+\pi^{2} I_{0}\left(u_{2 j-1}(x)\right)^{2}} .
$$


Note that $u_{j}(x)$ is found using Equation (4) with $n=2$. As $k$ is odd then there are $\left[\frac{k}{2}\right]+1$ integrals of the form Equation (13) and there are $\left[\frac{k}{2}\right]+1$ of the form Equation (14). Thus

$$
\begin{aligned}
& \int_{\gamma_{0}}+\int_{\Gamma_{1}}+\int_{\Gamma_{1}^{\prime}}+\cdots+\int_{\Gamma_{k+1}}+\int_{\Gamma_{k+1}^{\prime}} \\
& =-2 \pi i-2 i \sum_{j=1}^{\left[\frac{k}{2}\right]+1} \int_{a_{2 j-2}}^{a_{2 j-1}} \frac{1}{x} \mathrm{e}^{-\chi t} \Psi_{2 j-2}(r, x) \mathrm{d} x-2 \pi i \sum_{j=1}^{\left[\frac{k}{2}\right]+1} \int_{a_{2 j-1}}^{a_{2 j}} \frac{1}{x} \mathrm{e}^{-\chi t} \Psi_{2 j-1}(r, x) \mathrm{d} x .
\end{aligned}
$$

On the other hand, for $\int_{\gamma_{k+1}}$, we have that

$$
\left|\int_{\gamma_{k+1}}\right|<\int_{\alpha}^{\pi} \mathrm{e}^{R \operatorname{tcos}(\theta)} \frac{K_{0}\left(r \sqrt{\left(R^{k+1}\left(\frac{a_{1}}{R}+1\right)\left(\frac{a_{2}}{R}+1\right) \cdots\right)\left(\frac{a_{k-1}}{R}+1\right)\left(\frac{a_{k}}{R}+1\right)}\right)}{K_{0}\left(\sqrt{\left(R^{k+1}\left(\frac{a_{1}}{R}+1\right)\left(\frac{a_{2}}{R}+1\right) \cdots\right)\left(\frac{a_{k-1}}{R}+1\right)\left(\frac{a_{k}}{R}+1\right)}\right)} \mathrm{d} \theta,
$$

so, $\int_{\gamma_{k+1}}$ is 0 when $R \rightarrow \infty$ for $\delta$ sufficiently small. Similarly to $\int_{\gamma_{k+2}}$. Thus, using $\int_{\gamma_{0}}=-2 \pi i$, Equation (15) and Equation (16), we arrive to

$$
\begin{aligned}
f(t)= & +\frac{1}{\pi} \sum_{j=1}^{\left[\frac{k}{2}\right]+1} \int_{a_{2 j-2}}^{a_{2 j-1}} \frac{1}{x} \mathrm{e}^{-\chi t} \Psi_{2 j-2}(r, x) \mathrm{d} x \\
& +\sum_{j=1}^{\left[\frac{k}{2}\right]+1} \int_{a_{2 j-1}}^{a_{2 j}} \frac{1}{X} \mathrm{e}^{-\chi t} \Psi_{2 j-1}(r, x) \mathrm{d} x
\end{aligned}
$$

\section{Analytical Examples and Numerical Approximation}

In this section, we give some analytical examples corresponding to the exact solutions of the previous theorems and solve the integrals numerically for some particular cases.

Example 1. We consider that $k=1, n=2,0=a_{0}<a_{1}<a_{2}=\infty$ and $F(s)=\frac{1}{s} \sqrt{s\left(s+a_{1}\right)}$, then using $(2)$

$$
\begin{aligned}
f(t)= & \frac{1}{\pi} \sin \left(\frac{(0+1) \pi}{2}\right) \int_{0}^{a_{1}} \frac{1}{x} \mathrm{e}^{-x t} \sqrt{x\left(-x+a_{1}\right)} \mathrm{d} x \\
& +\frac{1}{\pi} \sin \left(\frac{(1+1) \pi}{2}\right) \int_{a_{1}}^{\infty} \frac{1}{x} \mathrm{e}^{-x t} \sqrt{x\left(x-a_{1}\right)} \mathrm{d} x \\
= & \frac{1}{\pi} \int_{0}^{a_{1}} \frac{1}{x} \mathrm{e}^{-x t} \sqrt{x\left(-x+a_{1}\right)} \mathrm{d} x .
\end{aligned}
$$

Furthermore, since

$$
\int_{0}^{a_{1}} \frac{1}{x} \mathrm{e}^{-x t} \sqrt{x\left(-x+a_{1}\right)} \mathrm{d} x=\frac{a_{1}}{2} \mathrm{e}^{-\frac{a_{1} t}{2}} \pi\left(I_{0}\left(\frac{a_{1} t}{2}\right)+I_{1}\left(\frac{a_{1} t}{2}\right)\right),
$$

with $I_{\alpha}$ is the modified Bessel function of order $\alpha$, then

$$
f(t)=\frac{a_{1}}{2} \mathrm{e}^{-\frac{a_{1} t}{2}}\left(I_{0}\left(\frac{a_{1} t}{2}\right)+I_{1}\left(\frac{a_{1} t}{2}\right)\right) .
$$


Example 2. We consider that $k=2, n=2,0=a_{0}<a_{1}<a_{2}<a_{3}=\infty$ and

$$
\begin{aligned}
F(s)=\frac{1}{\sqrt{s\left(s+a_{1}\right)\left(s+a_{2}\right)}}, \text { then using }(7) \\
\qquad \begin{aligned}
f(t)= & \frac{1}{\pi} \sin \left(\frac{(0+1) \pi}{2}\right) \int_{0}^{a_{1}} \mathrm{e}^{-x t} \frac{1}{\sqrt{x\left(-x+a_{1}\right)\left(-x+a_{2}\right)}} \mathrm{d} x \\
& +\frac{1}{\pi} \sin \left(\frac{(1+1) \pi}{2}\right) \int_{a_{1}}^{a_{2}} \mathrm{e}^{-x t} \frac{1}{\sqrt{x\left(x-a_{1}\right)\left(-x+a_{2}\right)}} \mathrm{d} x \\
& +\frac{1}{\pi} \sin \left(\frac{(2+1) \pi}{2}\right) \int_{a_{2}}^{\infty} \mathrm{e}^{-x t} \frac{1}{\sqrt{x\left(x-a_{1}\right)\left(x-a_{2}\right)}} \mathrm{d} x,
\end{aligned}
\end{aligned}
$$

so

$$
\begin{aligned}
f(t)= & \frac{1}{\pi} \int_{0}^{a_{1}} \mathrm{e}^{-x t} \frac{1}{\sqrt{x\left(-x+a_{1}\right)\left(-x+a_{2}\right)}} \mathrm{d} x \\
& -\frac{1}{\pi} \int_{a_{2}}^{\infty} \mathrm{e}^{-x t} \frac{1}{\sqrt{x\left(x-a_{1}\right)\left(x-a_{2}\right)}} \mathrm{d} x
\end{aligned}
$$

Example 3. We consider that $k=1, n=3, \quad m=2,0=a_{0}<a_{1}=\pi<a_{2}=\infty$ and $F(s)=\frac{1}{s} \frac{\sqrt[3]{s}}{\sqrt[2]{\left(s+a_{1}\right)}}$, then using $(8)$

$$
\begin{aligned}
f(t)= & \frac{1}{\pi} \sum_{j=0}^{\left[\frac{1}{2}\right]} \sin \left(\frac{(j+1) \pi}{3}-\frac{j \pi}{2}\right) \int_{a_{2 j}}^{a_{2 j+1}} \frac{1}{x} \mathrm{e}^{-x t} u_{2 j}(x) \mathrm{d} x \\
& +\frac{1}{\pi} \sum_{j=0}^{\left[\frac{1}{2}\right]} \sin \left(\frac{(j+1) \pi}{3}-\frac{(j+1) \pi}{2}\right) \int_{a_{2 j+1}}^{a_{2 j+2}} \frac{1}{x} \mathrm{e}^{-x t} u_{2 j+1}(x) \mathrm{d} x,
\end{aligned}
$$

so

$$
\begin{aligned}
f(t)= & \frac{1}{\pi} \sin \left(\frac{\pi}{3}\right) \int_{0}^{\pi} \frac{1}{x} \mathrm{e}^{-x t} \frac{\sqrt[3]{s}}{\sqrt{-x+\pi}} \mathrm{d} x \\
& +\frac{1}{\pi} \sin \left(\frac{-\pi}{6}\right) \int_{\pi}^{\infty} \frac{1}{x} \mathrm{e}^{-x t} \frac{\sqrt[3]{s}}{\sqrt{x-\pi}} \mathrm{d} x \\
= & \frac{3 \Gamma(5 / 6) t^{1 / 6}}{\pi}{ }_{1} F_{1}(1 / 2,7 / 6,-\pi t),
\end{aligned}
$$

where ${ }_{1} F_{1}$ is the hypergeometric function.

Example 4. We consider that $r>0, k=1, a_{1}=\frac{1}{\epsilon}$ and

$$
\begin{aligned}
& F(s)=\frac{1}{s} \frac{k_{0}(r \sqrt{s(s+1 / \epsilon)})}{k_{0}(\sqrt{s(s+1 / \epsilon)})} \text { then using }(9) \\
& \quad f(t)=1+\frac{1}{\pi} \int_{0}^{\frac{1}{\epsilon}} \frac{1}{x} \mathrm{e}^{-x t} \Psi_{0}(r, x) \mathrm{d} x+\int_{\frac{1}{\epsilon}}^{\infty} \frac{1}{x} \mathrm{e}^{-x t} \Psi_{1}(r, x) \mathrm{d} x
\end{aligned}
$$

where

$$
\Psi_{0}(r, x)=\frac{Y_{0}\left(u_{0}(x)\right) J_{0}\left(u_{0}(x) r\right)-J_{0}\left(u_{0}(x)\right) Y_{0}\left(u_{0}(x) r\right)}{J_{0}\left(u_{0}(x)\right)^{2}+Y_{0}\left(u_{0}(x)\right)^{2}},
$$




$$
\begin{gathered}
\Psi_{1}(r, x)=\frac{I_{0}\left(u_{1}(x)\right) K_{0}\left(u_{1}(x) r\right)-K_{0}\left(u_{1}(x)\right) I_{0}\left(u_{1}(x) r\right)}{K_{0}\left(u_{1}(x)\right)^{2}+\pi^{2} I_{0}\left(u_{1}(x)\right)^{2}}, \\
u_{0}(x)=\sqrt{x(-x+1 / \epsilon)} \text { and } u_{1}(x)=\sqrt{x(x-1 / \epsilon)} .
\end{gathered}
$$

On the other hand, we solve the integrals numerically corresponding to the exact solutions of Theorems 1, 2, 3 and 4 then we compare versus Stehfest numerical method. This method consist in numerically finding the Laplace inverse transform of the $F(s)$ using:

$$
f(t)=\frac{\log (2)}{t} \sum_{i=1}^{n_{1}} g(i) F\left(\frac{\log (2)}{t} i\right)
$$

where

$$
g(i)=(-1)^{\frac{n_{1}}{2}+i} \sum_{k=\left[\frac{i+1}{2}\right]}^{\min \left\{i, \frac{n_{1}}{2}\right\}} \frac{k^{\frac{n_{1}}{2}}(2 k) !}{\left(\frac{n_{1}}{2}-k\right) ! k !(k-1) !(i-k) !(2 k-i) !} .
$$

In Figure 2(a) and Figure 2(b) we observed in red color Equations ((2) and

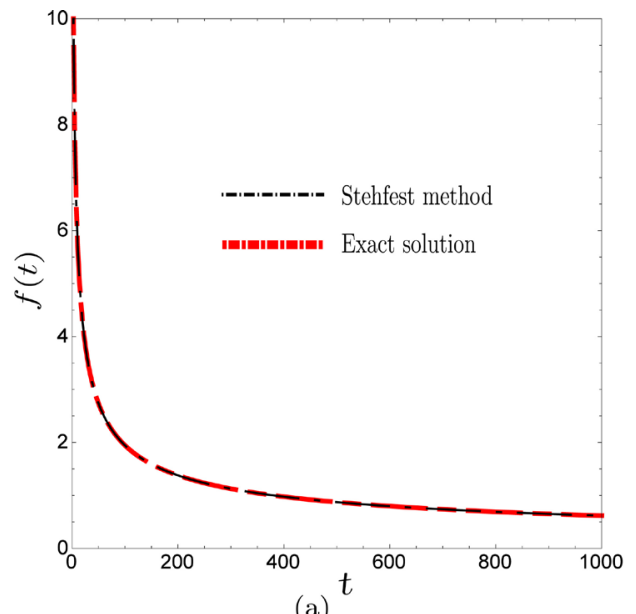

(a)

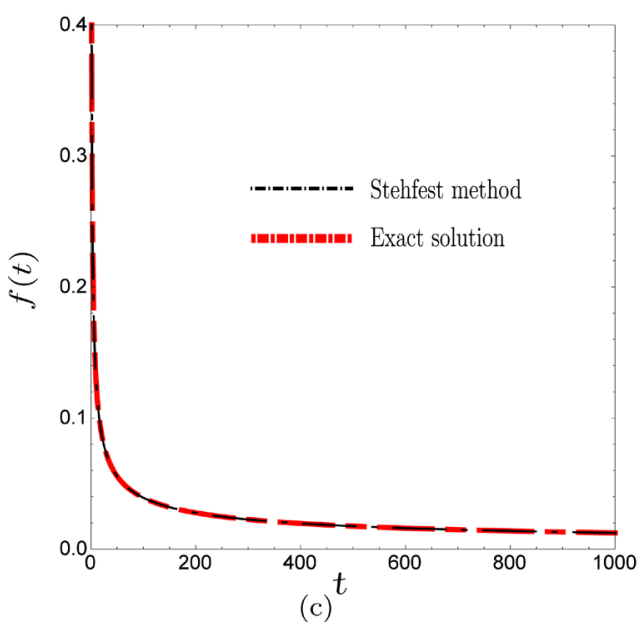

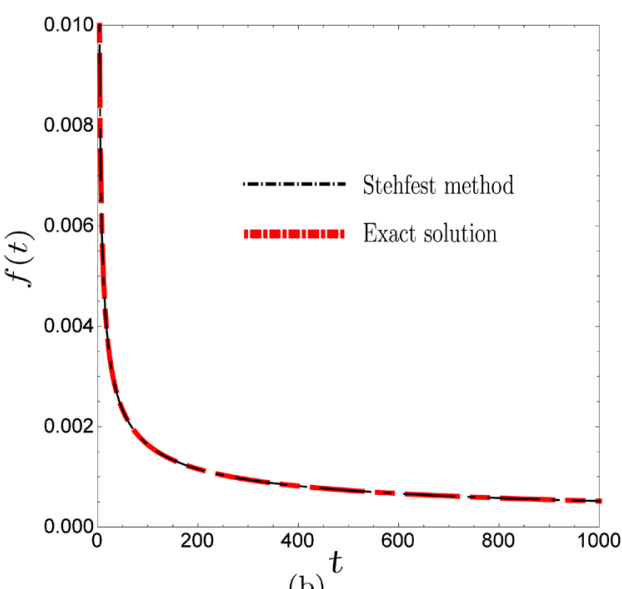

(b)

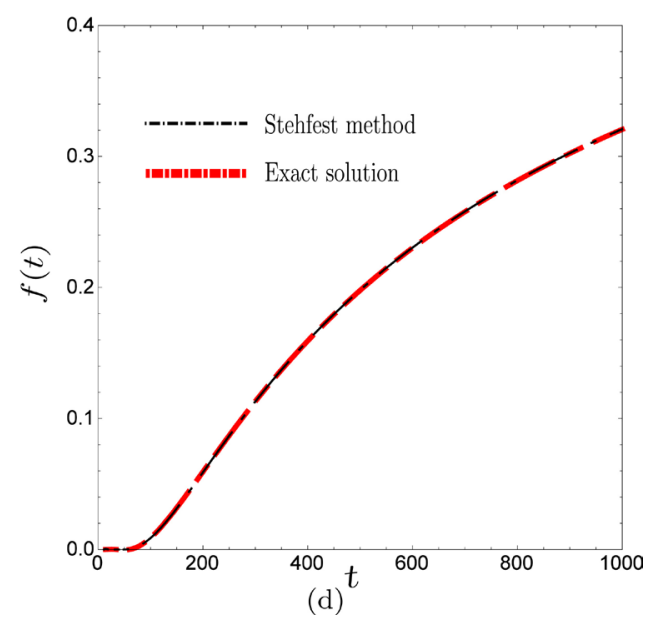

Figure 2. Comparison of the exact solution (2), (7), (8), (9) with parameters $n=2, k=5, a_{1}=1$, $a_{2}=\pi, a_{3}=5, a_{4}=\mathrm{e}^{2}, a_{5}=10, r=2, m=2$ and the Stehfest numerical method with $n_{1}=14$. 


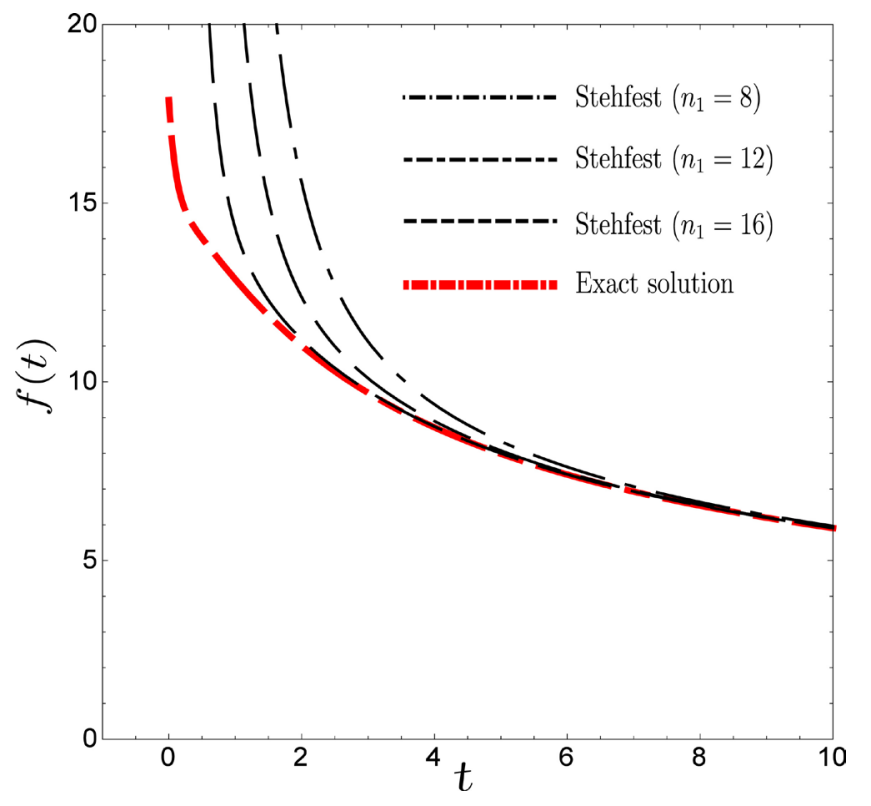

Figure 3. Comparison of the exact solution (2) with paramteters $n=2, k=5, a_{1}=1$, $a_{2}=\pi, a_{3}=5, a_{4}=\mathrm{e}^{2}, a_{5}=10$ and Stehfest numerical method for different $n_{1}$, this is for $t$ small.

(7)) respectively with parameters $n=2, k=5, a_{1}=1, a_{2}=\pi, a_{3}=5$, $a_{4}=\mathrm{e}^{2}$ and $a_{5}=10$. In Figure 2(c) we observed Equation (8) in red color with the same previous parameters in conjunction with $m=2$. In Figure 2(d) we observed Equation (9) in red color with the same previous parameters in conjunction with $r=2$. On the other hand, in all plots of Figure 2 Stehfest numerical method is shown in black color with $n_{1}=14$ and functions $F(s)$ corresponding to the aforetmentioned parameters for each cases. As expected in all cases the exact solution of the theorems proposed here coincide with Stehfest numerical method for large $t$ values, however the stephens method does not approach well near the discontinuities of the function, for example in Figure 3 we see in red color the same exact solution of Figure 2(a) for small $t$ values, and in black color the Stehfest numerical method for different $n_{1}$. In this figure is shown that if $n_{1}$ increase then Stehfest numerical method approximates better to exact solution near zero but still the approximation is bad, this is because Stehfest numerical method does not give a good approximation near points where the functions are discontinuous (see [10]).

\section{Conclusion}

In this work, we solved the inverse Laplace transform for multivalued functions that involving the $n$th root of a product of linear factors, we show that results are correct and also give analytical and numerical examples. The numerical examples were compared with Stehfest numerical method, concluding that the curves coincide for values far from the discontinuities of the solution, while for values close to the discontinuity the Stehfest numerical method does not have good approximation. 


\section{References}

[1] Arciga, M.P., Ariza, F.J., Sanchez, J. and Salmeron, U. (2016) Fractional Stochastic Heat Equation on the Half-Line. Applied Mathematical Sciences, 10, 3095-3105. https://doi.org/10.12988/ams.2016.69243

[2] Di Paola, M., Pirrotta, A. and Valenza, A. (2011) Visco-Elastic Behavior through Fractional Calculus: An Easier Method for Best Fitting Experimental Results. $\mathrm{Me}$ chanics of Materials, 43, 79-806. https://doi.org/10.1016/j.mechmat.2011.08.016

[3] Ekoue, F., Halloy A.F., Gigon, D., Plantamp, G. and Zajdman, E. (2013) Maxwell-Cattaneo Regularization of Heat Equation. International Scholarly and Scientific Research and Innovation, 7, 772-775.

[4] Golghanddashti, H. (2011) A New Analytically Derived Shape Factor for Gas-Oil Gravity Drainage Mechanism. Journal of Petroleum Science and Engineering, 77, 18-26. https://doi.org/10.1016/j.petrol.2011.02.004

[5] Huan, X., Hai,Q. and Xiao, J. (2013) Fractional Cattaneo Heat Equation in a Semi Infinite Medium. Chinese Physics B, 22, 014401-1-014401-6. https://doi.org/10.1088/1674-1056/22/1/014401

[6] Shu, L. and Bing, C. (2016) Generalized Variational Principles for Heat Conduction Models Based on Laplace Transforms. International Journal of Heat and Mass Transfer, 103, 1176-1180. https://doi.org/10.1016/j.ijheatmasstransfer.2016.08.065

[7] Rehbinder, G. (1989) Darcyan Flow with Relaxation Effect. Applied Scientific Research, 46, 45-72. https://doi.org/10.1007/BF00420002

[8] Hernndez, D., Nez, M. and Velasco, J. (2013) Telegraphic Double Porosity Models for Head Transient Behavior in Naturally Fractured Aquifers. Water Resources Research, 49, 4399-4408. https://doi.org/10.1002/wrcr.20347

[9] Wu, Y., Ehlig-Economides, C., Qin, G., Kang, Z., Zhang, W., Ajayi, B. and Tao, Q. (2007) A Triple-Continuum Pressure-Transient Model for a Naturally Fractured Vuggy Reservoir. Lawrence Berkeley National Laboratory, Berkeley, CA. https://doi.org/10.2118/110044-MS

[10] Stehfest, H. (1970) Algorithm 368: Numerical Inversion of Laplace Transforms [d5]. Communications of the ACM, 13, 47-49. https://doi.org/10.1145/361953.361969

[11] Lfqvist, T. and Rehbinder, G. (1993) Transient Flow towards a Well in an Aquifer including the Effect of Fluid Inertia. Applied Scientific Research, 51, 611-623. https://doi.org/10.1007/BF00868003

[12] Gradshteyn, I.S. and Ryzhik, I.M. (2014) Table of Integrals, Series, and Products. Academic Press, New York.

[13] Abramowitz, M. and Stegun, I.A. (1964) Handbook of Mathematical Functions: With Formulas, Graphs, and Mathematical Tables. Courier Corporation, New York.

[14] Carslaw, H.S. and Jaeger, J.C. (1941) Operational Methods in Applied Mathematics, Dover, New York. 
Submit or recommend next manuscript to OALib Journal and we will provide best service for you:

- Publication frequency: Monthly

- 9 subject areas of science, technology and medicine

- Fair and rigorous peer-review system

- Fast publication process

- Article promotion in various social networking sites (LinkedIn, Facebook, Twitter, etc.)

- Maximum dissemination of your research work

Submit Your Paper Online: Click Here to Submit

Or Contact service@oalib.com 